Hautarzt 2015 - 66:303-304

DOI 10.1007/s00105-015-3625-x

Online publiziert: 26. März 2015

๑) Springer-Verlag Berlin Heidelberg 2015

\author{
M. Hertl ${ }^{1}$. J.M. Baron ${ }^{2}$ \\ ${ }^{1}$ Klinik für Dermatologie und Allergologie, Philipps Universität, Marburg, Deutschland \\ ${ }^{2}$ Klinik für Dermatologie und Allergologie - Hautklinik, RWTH, Aachen, Deutschland
}

\title{
Prof. Dr. Hans Merk zum 65. Geburtstag
}

ergologie (eigentlich Andrologie!) die klinische Allergologie der Kölner Universitätshautklinik ganz wesentlich geprägt. In der nationalen und internationalen Allergologie ist er seit Jahrzehnten präsent und hoch geachtet als langjähriger Herausgeber und Ehrenherausgeber von Skin Pharmacology, Mitherausgeber des Allergo Journal, dermatotoxikologischer Lehrbücher und nicht zuletzt auch aufgrund seiner berufspolitischen Aktivitäten, $u$. a. als Präsident des AeDA (Ärzteverband Deutscher Allergologen).

Sein wissenschaftlicher Schwerpunkt Allergologie hat ihn international berühmt gemacht. Durch seine profunde Kenntnis der Dermatopharmakologie und -toxikologie hat er neben den breiter erforschten immunologischen Mechanismen von Arzneimittelallergien und allergischer Kontaktdermatitis eine wesentliche Grundlage für das Verständnis der Immunogenität von Haptenen gelegt. Wegweisend sind seine Arbeiten zum Fremdstoffmetabolismus kleinmolekularer Substanzen, die vor allem als Medikamente bzw. Kontaktallergene klinisch relevant sind. Jeder in der deutschen Dermatologie verbindet mit HFM (Hans F. Merk) ein weiteres Akronym: CYP450 (Cytochrom P450)! Hervorzuheben sind die Bemühungen von Prof. Merk, den Lymphozytentransformationstest als zelluläre In-vitro-Diagnostik bei kutanen Arzneireaktionen zu etablieren. Dieser war unter anderem Grundlage für die Identifizierung arzneimittelspezifischer CD8 ${ }^{+}$-T-Zellen und damit für ein neues Verständnis zellulärer Immunmechanismen bei Arzneimittelallergien. Ein wesentlicher Aachener Beitrag zur Allergieforschung war die Identifizierung epidermaler Transporterproteine, die für die perkutane Aufnahme von Haptenen wesentlich sind. Ferner ist Prof. Merk international anerkannt für seine Arbeiten zum Retinoidstoffwechsel und zur Charakterisierung beruflich bedingter allergologischer und nicht allergologischer Hauterkrankungen. Seine wissenschaftliche Arbeit wurde u. a. mit dem Oskar-Gans-Förderpreis der Deutschen Dermatologischen Gesellschaft und dem Herbert Herxheimer-Förderpreis der Deutschen Gesellschaft für Allergie- und Immunitätsforschung ausgezeichnet. Seit 2000 ist Prof. Merk Mitglied der Deutschen Akademie der Wissenschaften, Leopoldina.

\section{Werdegang und berufliche Entwicklung}

In Wuppertal geboren, ist Hans Merk rheinisch geprägt dank der Schulzeit in Leverkusen und dem Studium der Pharmazie und später Medizin in Düsseldorf. An der toxikologisch ausgerichteten Düsseldorfer Hautklinik begann er seine klinische Ausbildung zum Dermatologen und wechselte 1982 nach Köln, seiner eigentlichen „Heimatstadt des Herzens“.

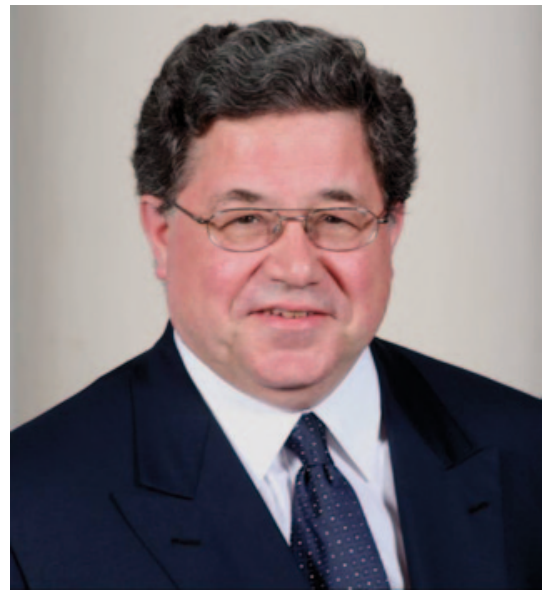

Abb. 1 ॥ Prof. Dr. Hans F. Merk 


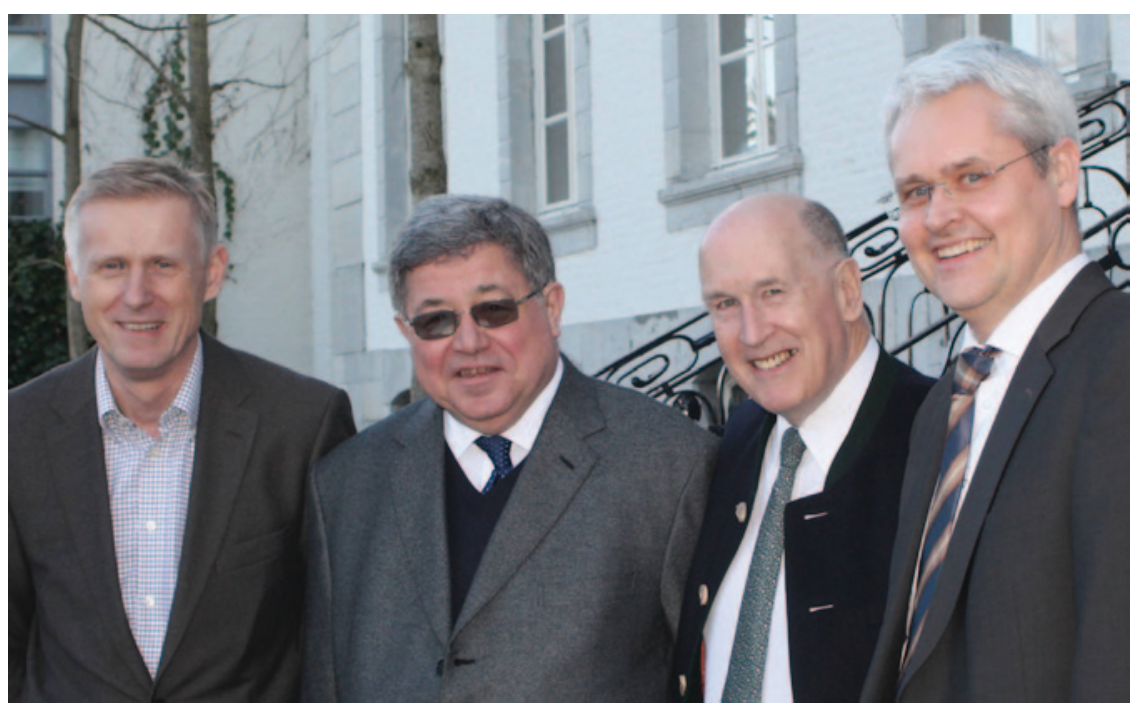

Abb. 2 A Abschiedssymposium am 21.02.2015 in Aachen: Profs. Michael Hertl, Hans F. Merk, David Bickers und Jens M. Baron (v.l.n.r.)

Nur eines passt bei Hans Merk nicht zum Vollblutrheinländer: Dem feucht-fröhlichen Frohsinn des rheinischen Karnevals kann er nicht viel abgewinnen und entgeht dem Trubel regelmäßig durch die Flucht in die USA zu seinem Freund David Bickers. Diese Zeiten hat er auch genutzt für mehrwöchige Forschungsaufenthalte in den Laboren der Hautkliniken in Cleveland und New York.

Nach 12 Jahren an der Kölner Hautklinik erhielt Prof. Merk 1994 den Ruf auf das Ordinariat für Dermatologie und Venerologie in der Kaiserstadt Aachen. In der nun bald 100-jährigen Historie der Aachener Dermatologie als eigenständiger Abteilung ist Prof. Merk sicherlich der am stärksten wissenschaftlich ausgerichtete Klinikdirektor. Die zunächst städtische Hautklinik wurde von 1918 bis 1947 von Friedrich H.H. Paulus, von 1947 bis 1962 von Philipp Keller und 1962 von Aloys Greither geleitet. Im Jahr 1966 wurde die Hautklinik Teil des neu gegründeten Universitätsklinikums unter der Direktion von Walther Gahlen (1962-1977) sowie nachfolgend Wolf Meinhof (19771994). Somit ist es auch ein wesentliches Verdienst von Prof. Merk, dass die Aachener Hautklinik in der "Szene" uneingeschränkt als Wissenschaftsstandort wahrgenommen wird.

\section{Lehrer und Mentor}

Last but not least: Prof. Hans Merk ist seinen Schülern immer ein engagierter Mentor gewesen und hat durch sein persönliches Wirken viele junge Medizinerinnen und Mediziner für die Dermatologie gewinnen können. Als ehemalige Doktoranden von Prof. Merk erinnern wir uns immer noch gerne an die "Anfänge": umfängliche, freundliche und interessante Gespräche, viele Anregungen für die wissenschaftliche Arbeit ohne Zwänge hinsichtlich der praktischen Umsetzung - und vor allem das Gefühl, von einem etablierten Professor ernst genommen zu werden! Prof. Merk war immer supportiv und hat viele Wege für die weitere Entwicklung seiner Schüler geebnet, insbesondere auf der Basis seiner engen Freundschaft mit Prof. Dr. David Bickers an der Case Western Reserve University in Cleveland und dann später an der Columbia University in New York (『Abb. 2). Einige seiner Schüler haben auf diese Weise auch ihren Weg in die akademische Dermatologie gefunden und denken dankbar an diese Zeit zurück. Prof. Merk ist Gründungsmitglied der BAER (Bicontinental Association of Education and Research in Dermatology), die ganz wesentlich den Austausch jun- ger, forschungsorientierter Dermatologen zwischen Deutschland und den USA gefördert hat. Man kann es auch so sagen: Prof. Merk hat wesentlich zur transatlantischen Vernetzung der deutschen Dermatologie beigetragen. Seine Unterstützung war immer selbstlos, sein Auftreten zurückhaltend bescheiden, unprätentiös, nie selbstherrlich oder eitel! Damit hat er auf seine ruhige, nie in den Vordergrund drängende, feine Art sehr viel für die deutsche Dermatologie geleistet!

\section{Würdigung und Glückwünsche}

Hans Merk besitzt eine Lebensphilosophie der besonderen Art mit einer gesunden Distanz zu den Unwägsamkeiten des Alltags, die das Leben einfacher macht. Seine Annotationen sind meist kurz und treffend, ohne zu verletzen. Als Beispiel sein Kommentar zur üppigen Weihnachtsdekoration einer engagierten Mitarbeiterin der Hautklinik: „Guter Wille ist das Gegenteil von gutem Geschmack" (frei nach Oskar Wilde).

Lieber Hans Merk, wir, Deine Schülerinnen und Schüler, sind Dir sehr dankbar für Deine Unterstützung und Deine vorgelebte Freude an der Dermatologie in allen ihren Facetten! - Wir wünschen Dir, dass Du weiterhin mit Deiner liebenswürdigen Art unser Fach auf nationaler und internationaler Ebene bereicherst, und freuen uns auf viele gemeinsame Stunden mit Dir! Bleib gesund und uns lange erhalten!

Im Namen Deiner Schülerinnen und Schüler!

Michael Hertl und Jens Baron

\section{Korrespondenzadresse}

Prof. Dr. M. Hertl

Klinik für Dermatologie und Allergologie

Philipps Universität

Baldinger Str.

35043 Marburg

hertl@med.uni-marburg.de 